\title{
Stable Partitions in Many Division Problems: The Proportional and the Sequential Dictator Solutions
}

\author{
Gustavo Bergantiños ${ }^{1}$, Jordi Massó ${ }^{2}$, Inés Moreno de Barreda ${ }^{3}$ and \\ Alejandro Neme ${ }^{4}$
}

July 2014

\begin{abstract}
We study how to partition a set of agents in a stable way when each coalition in the partition has to share a unit of a perfectly divisible good, and each agent has symmetric single-peaked preferences on the unit interval of his potential shares. A rule on the set of preference profiles consists of a partition function and a solution. Given a preference profile, a partition is selected and as many units of the good as the number of coalitions in the partition are allocated, where each unit is shared among all agents belonging to the same coalition according to the solution. A rule is stable at a preference profile if no agent strictly prefers to leave his coalition to join another coalition and all members of the receiving coalition want to admit him. We show that the proportional solution and all sequential dictator solutions admit stable partition functions. We also show that stability is a strong requirement that becomes easily incompatible with other desirable properties like efficiency, strategy-proofness, anonymity, and non-envyness.
\end{abstract}

Journal of Economic Literature Classification Number: D71.

Keywords: Division Problem, Symmetric Single-peaked Preferences, Stable Partition.

\footnotetext{
${ }^{1}$ Research Group in Economic Analysis. Facultad de Económicas, Universidad de Vigo. 36310, Vigo (Pontevedra), Spain. E-mail: gbergant@uvigo.es

${ }^{2}$ Universitat Autònoma de Barcelona and Barcelona GSE. Departament d'Economia i d'Història Econòmica, 08193, Bellaterra (Barcelona), Spain. E-mail: jordi.masso@uab.es

${ }^{3}$ Nuffield College, Oxford University, New Road Oxford OX1 1NF, United Kingdom. E-mail: ines.morenodebarreda@economics.ox.ac.uk.

${ }^{4}$ Instituto de Matemática Aplicada de San Luis. Universidad Nacional de San Luis and CONICET. Ejército de los Andes 950. 5700, San Luis, Argentina. E-mail: aneme@unsl.edu.ar
} 


\section{Introduction}

Consider the division problem faced by a set of agents who have to share a unit of an homogeneous and perfectly divisible good. For instance, a group of agents participate in an activity that requires a fixed amount of labor measured in units of time. Given a wage, classical monotonic and quasiconcave preferences on the set of bundles of money and leisure generate single-peaked preferences on the set of potential shares, where the best share is the amount of working time associated to the optimal bundle and in both sides of the best share the preference is strictly monotonic, decreasing at its right and increasing at its left. Similarly, a group of agents join a partnership to invest in a project (an indivisible bond with a face value, for example) that requires a fixed amount of money, neither more nor less. Their risk attitudes and wealth induce single-peaked preferences on the amount to be invested. As in the previous examples, there are other social choice settings for which the division problem appears as its reduced problem (see for example, Barberà and Jackson (1995)).

A solution is a family of mappings that select for each instance of division problem (a set of agents and their single-peaked preferences) a vector of shares, one for each agent. But for most single-peaked preference profiles, the sum of the best shares will be either larger or smaller than the total amount to be allocated. A positive or negative rationing problem emerges depending on whether the sum of the best shares exceeds or falls short of the fixed amount. Sprumont (1991) started a large literature characterizing solutions in terms of alternative sets of properties. These solutions differ on the underlying principles guiding how the rationing problem has to be solved. ${ }^{1}$

In this paper, we study the division problem when the good to be allocated also comes with fixed amounts but now agents may share several units, whose number is endogenous because it may depend on agents' preferences. Consider for example a group of entrepreneurs examining several business opportunities. Each entrepreneur is willing to devote himself to at most one of those business opportunities and as before, their risk attitudes and wealth induce single-peaked preferences on the amount to be invested. We let agents partition themselves into coalitions in such a way that agents in each coalition will have to share one and only one unit of the good. We want to emphasize that the class of real-life examples that we want to model have to have the feature that every coalition of the partition, no matter what its composition is, gets a full unit to allocate among its members; for instance, when a set of agents who want to invest some of their savings in a particular indivisible bond, no coalition is able to buy strictly less than the face value of the bond. An allocation is a pair consisting of a partition of the set of agents and a vector of allotments specifying for each coalition in the partition a vector of shares, one for each agent in the coalition, whose components add up to one unit. A rule is a mapping that selects for each profile of single-peaked preferences an allocation. Thus, a rule can be decomposed into two procedures. For each profile of single-peaked preferences, the first procedure is a function that selects a partition of the set of agents while the second procedure is a solution to be applied to the subprofile of single-peaked preferences of the agents in each coalition of the partition. We restrict ourselves to second procedures that select the allotment by means of a unique solution applied to each rationing problem faced by each coalition in the partition. This restriction implies that the

\footnotetext{
${ }^{1}$ For axiomatic characterizations of solutions see for example Barberà, Jackson, and Neme (1997), Ching (1992, 1994), Dagan (1996), Ehlers (2002a, 2002b), Herrero and Villar (2000), Schummer and Thomson (1997), Sönmez (1994), and Thomson (1994, 1995, 1997, 2003).
} 
same principles are used across coalitions and it can be interpreted as a consistency requirement. Thus, a rule can be identified with a partition function (mapping single-peaked preference profiles into partitions of the set of agents) and a solution (to be applied to each coalition of the selected partition).

Our main concern in this paper is the stability of rules. ${ }^{2}$ Specifically, fix a solution. We want to know whether there exists a partition function that, together with the fixed solution, constitute a stable rule. Our notion of stability is based on the principle that the allocations proposed by the rule have to be voluntarily accepted by the agents in the following sense. Consider a rule and a profile of single-peaked preferences. Apply the rule to the profile, thereby obtaining a partition and a vector of allotments. Take an agent in a coalition and another coalition (which may be empty), and suppose that (1) the agent wants to leave his original coalition to join the other one because the share assigned to him by the solution applied to the subprofile of preferences of the agents in the new coalition is strictly preferred to his former share, and (2) all agents of the receiving coalition want to admit the agent because the shares assigned to them by the solution applied to the subprofile of preferences of the agents in the new coalition are weakly preferred to their respective former shares. In this case, the original chosen allocation would be unstable at the profile to which the rule has been applied. A rule is stable if it chooses stable allocations at each profile of single-peaked preferences. Three remarks are in order. First, to generate an instability we are requiring that the moving agent has to obtain a strictly preferred share while the agents of the receiving coalition have to obtain a weakly preferred share. This captures the idea that to move from one coalition to another (the origin of the instability) requires a bit more than just to admit a new member in the coalition. Second, instabilities are generated only by one agent moving to a new coalition. In this case, the needed coordination among agents to fulfill the instability is minimal compared with the coordination needed if non-singleton subcoalitions would be allowed to change coalitions. Third, the receiving coalition may be empty, in which case the instability would be produced only by the agent that by leaving his current coalition could be strictly better off; i.e., the agent would strictly prefer the full share of one unit of the good to the share he had been assigned in his original coalition.

In a similar setting Gensemer et al $(1996,1998)$ study another concept of stability that they call "migration equilibrium". Agents with single-peaked preferences are partitioned into several local economies, each of which has an endowment that is allocated among its participants following a given solution. A migration equilibrium requires that no agent will be better off by leaving his economy to join another. They show that when the solution applied to each local economy is well behaved there might not exist a migration equilibria. ${ }^{3}$ Note that the receiving economy cannot ban the arrival of a new agent and hence the migration equilibrium is a stronger stability condition

\footnotetext{
${ }^{2}$ Kar and Kibris (2008) consider the efficiency of such rules in a setting where the number of units to share is fixed rather than endogenous. They show that for the domain of single-peaked preferences and for well behaved solutions (efficient, non dictatorial, strategy proof, resource monotonic and consistent), it is not possible to find a partition such that the final allocation is efficient.

${ }^{3}$ In particular, in Gensemer et al (1996) they show that a migration equilibrium might fail to exists if the solution applied to the local economies satisfies two of the following three properties: Pareto efficiency, strategy proofness and no-envy. In Gensemer et al (1998) they show that a migration equilibrium might fail to exists whenever the solution applied to the local economies is either the Proportional, the Sequential Dictator, the Uniform or the Egalitarian rules.
} 
than the one studied in this paper. Which stability concept to apply depends on the applications. In an environment with a small number of agents with decision power such as in joint ventures, our concept is more appealing whereas for movements across countries or big societies the migration equilibrium is the one to be considered. ${ }^{4}$

We found that in general, finding partition functions for well-known and simple solutions, to constitute together stable rules, is not an easy task. Indeed, it may become extremely complex in the general setting of the division problem. Thus, we have simplified the problem by assuming that agents' single-peaked preferences are in addition symmetric. ${ }^{5}$ A single-peaked preference is symmetric if the following additional condition holds: a share is strictly preferred to another one if and only if the former is strictly closer to the best share. Observe that in many applications the linear order structure on the set of potential shares, relative to which single-peakedness is defined, conveys to agents' preferences more than just an ordinal content. Often, an agent's preference on the set of shares is responsive also to the notion of distance, embedding to the preference its corresponding property of symmetry (see Massó and Moreno de Barreda (2011) for the use of symmetric single-peaked preferences in the context of selecting a public good, as in Moulin (1980)). The use of symmetric single-peaked preferences has the additional advantage that, without loss of generality, the domain of the rule is the set of vectors of best shares, instead of the set of profiles of full preferences.

Our main results establish that, provided that agents' preferences are symmetric single-peaked, the proportional solution (Proposition 1) and all sequential dictator solutions (Proposition 2) have the property that for each one of them there exists a partition function that, together with the corresponding solution, constitute a stable rule. ${ }^{6}$ The proportional solution of the division problem assigns to each agent, given a vector of agents' best shares, a share that is equal to his best share divided by the sum of all the best shares. Remember that the solution is applied to each coalition in the partition selected by the partition function at the vector of agents' best shares. Given an ordering on the set of agents, the sequential dictator solution associated with this ordering, and applied to a vector of agents' best shares, let each agent, except the last one, choose sequentially (following the ordering) his share of what is left of the good (if anything) by his predecessors. The last agent in the ordering gets the remainder. Observe that (i) each ordering on the set of agents define a different solution of the division problem, and (ii) the order is fixed and used in each of the coalitions selected by the partition function at the same vector of agents' best shares. The proofs of the two results are constructive and proceed by induction on the number of agents. In addition, we exhibit examples showing that for both rules stability is a strong requirement incompatible with many other desirable properties like efficiency, strategy-proofness, anonymity, and non-envyness.

\footnotetext{
${ }^{4}$ Conley and Konishi (2000) propose another stability concept, "migration-proof Tiebout equilibrium", which is weaker than the migration equilibrium. They show that for sufficiently large populations of homogeneous agents, a migration-proof Tiebout equilibrium exists, is unique and asymptotically efficient. See also Sertel (1992) for an alternative notion of stability and efficiency of partitions, in which a membership property rights code is used in an abstract setting.

${ }^{5}$ Kar and Kibris (2008) show that in the domain of symmetric singled-peaked preferences, and when the number of units of the good to be shared is fixed, whenever a (local) solution is efficient, there exists a partition such that the final allocation is efficient. At the end of the paper we further discuss on the importance of assuming that agents have symmetric single-peaked preferences.

${ }^{6}$ Note that for both the proportional solution and the sequential dictator solution a migration equilibrium (Gensemer et al $(1996,1998))$ might fail to exists even when we restrict the preferences to be symmetric single-peaked.
} 
We also show that there are simple solutions for which there do not exist partition functions that together constitute stable rules. In particular, we exhibit an example of a vector of weights (one for each agent) and a profile of preferences with the property that there is no partition function that, together with the corresponding weighted proportional solution, constitute a stable rule.

Amorós (2002), Adachi (2010), and Morimoto, Serizawa, and Ching (2013) study also multidimensional extensions of Sprumont (1991)'s division problem. They extend the uniform solution of a division problem to many division problems (Amorós (2002) does it for problems with only two agents). Their approach is different to ours because they consider problems where the goods to be allocated may be different and each agent has preferences on vectors of his potential shares (one for each different good). Their main contribution is to extend and axiomatically characterize the uniform solution to the multiple goods setting.

The paper is organized as follows. In Section 2 we introduce the model. In Section 3 we state and prove our main results. In Section 4 we present some final comments.

\section{Preliminaries}

Agents are indexed by the elements of a finite set $N=\{1, \ldots, n\}$, where $n \geq 1$. They have to partition themselves in such a way that the agents of each coalition of the partition have to share one unit of a perfectly divisible good (as in Sprumont (1991)). Let $\Pi$ denote the set of partitions of $N$. Given $\pi=\left\{S_{1}, \ldots, S_{K}\right\} \in \Pi$ and $i \in N$, let $S_{\pi}(i)$ denote the set $S_{k} \in \pi$ such that $i \in S_{k}$. An allocation is a pair $(\pi, x)$ where $\pi=\left\{S_{1}, \ldots, S_{K}\right\} \in \Pi$ is a partition and $x=\left(x_{1}, \ldots, x_{n}\right) \in[0,1]^{n}$ is a vector of allotments such that, for each $k=1, \ldots, K$,

$$
\sum_{i \in S_{k}} x_{i}=1
$$

Let $A$ be the set of allocations. We assume that each agent $i \in N$ has a complete and transitive preference relation $R_{i}$ on the set of his potential shares [0,1]. Let $P_{i}$ and $I_{i}$ be its associated strict and indifference relations, respectively. Namely, for each pair $x_{i}, y_{i} \in[0,1], x_{i} P_{i} y_{i}$ if and only if $y_{i} R_{i} x_{i}$ does not hold, and $x_{i} I_{i} y_{i}$ if and only if $x_{i} R_{i} y_{i}$ and $y_{i} R_{i} x_{i}$ hold. In addition, we assume that $R_{i}$ is symmetric single-peaked; that is, there exists the best share $b_{i} \in[0,1]$ (i.e., $b_{i} P_{i} x_{i}$ for all $\left.x_{i} \in[0,1] \backslash\left\{b_{i}\right\}\right)$ and $x_{i} P_{i} y_{i}$ if and only if $\left|x_{i}-b_{i}\right|<\left|y_{i}-b_{i}\right|$. Therefore, we can identify a profile of preference relations $R=\left(R_{1}, \ldots, R_{n}\right)$ by the vector of their corresponding best shares $b=\left(b_{1}, \ldots, b_{n}\right)$, which we call a profile. The set of all profiles is $[0,1]^{n}$. Given a non-empty subset $S \subseteq N$, we denote its cardinality by its lower case representation, i.e., $s=\# S$. Given a profile $b \in[0,1]^{n}$ and a non-empty subset $S \subseteq N$ we denote by $b_{S}=\left(b_{i}\right)_{i \in S} \in[0,1]^{s}$ the subprofile of best shares of agents in $S$.

Given a non-empty subset of agents $S \subseteq N$, a solution for $S$ is a function $f^{S}:[0,1]^{s} \rightarrow[0,1]^{s}$ that selects for each subprofile an allotment for $S$; namely, for each $b_{S} \in[0,1]^{s}, f^{S}\left(b_{S}\right) \in[0,1]^{s}$ has the property that

$$
\sum_{i \in S} f_{i}^{S}\left(b_{S}\right)=1
$$

A solution $f$ is a family $\left\{f^{S}\right\}_{S \subseteq N}$, where each $f^{S}$ is a solution for $S .^{7}$ Given a solution $f$, a partition $\pi$, and a profile $b$, denote by $f(\pi, b)=\left(f_{i}(\pi, b)\right)_{i \in N}$ the following vector of allotments:

\footnotetext{
${ }^{7}$ Note that the definition of solution allows for each subset $S \subseteq N$ to have its own distinct solution $f^{S}$. As we
} 
for each $i \in N$,

$$
f_{i}(\pi, b)=f_{i}^{S_{\pi}^{(i)}}\left(b_{S_{\pi}(i)}\right) .
$$

A partition function is a mapping $\mu:[0,1]^{n} \rightarrow \Pi$ that selects, for each profile $b \in[0,1]^{n}$, a partition $\mu(b) \in \Pi$.

A rule is a function $\Phi:[0,1]^{n} \rightarrow A$ selecting, for each profile $b \in[0,1]^{n}$, an allocation $\Phi(b)=$ $(\pi, x) \in A$. Given a partition function $\mu$ and a solution $f, \Phi=(\mu, f)$ is the rule that for all $b \in[0,1]^{n}$, selects the allocation $\Phi(b)=(\mu(b), f(\mu(b), b))$. We can also apply a rule to a subprofile in the obvious way.

In the next section we will focus on the stability of some rules. We now define this property. Consider a rule. Given a profile, apply the rule to the profile, thereby obtaining a partition and a vector of allotments. Now, imagine that an agent moves to another coalition in the partition and, after applying the solution to this new subset, he obtains a strictly better share and all former members of this receiving coalition (which may be empty) are at least as well as they were before. Then, the rule would not be stable at the profile. To define formally a stable rule we have to describe, given a partition, an agent and a coalition receiving this agent, the new partition after the agent moves from the former coalition to the new one. Given $\pi=\left\{S_{1}, \ldots, S_{K}\right\}, i \in N$, and $k \in\{1, \ldots, K\}$ define

$$
\pi_{-i, k}= \begin{cases}{\left[\pi \backslash\left(\left\{S_{\pi}(i)\right\} \cup\left\{S_{k}\right\}\right)\right] \cup\left\{\left\{S_{\pi}(i)\right\} \backslash\{i\}\right\} \cup\left\{S_{k} \cup\{i\}\right\}} & \text { if } S_{k} \neq S_{\pi}(i) \\ {\left[\pi \backslash\left\{S_{\pi}(i)\right\}\right] \cup\left\{\left\{S_{\pi}(i)\right\} \backslash\{i\}\right\} \cup\{i\}} & \text { if } S_{k}=S_{\pi}(i) .\end{cases}
$$

Observe that if $i \notin S_{k}$ then $\pi_{-i, k}$ is the new partition where all coalitions remain the same except that $S_{\pi}(i)$ looses $i$ and $S_{k}$ gains $i$. But if $i \in S_{k}$ then $\pi_{-i, k}$ is the new partition where all coalitions remain the same except that $S_{\pi}(i)$ looses $i$ and $\{i\}$ is itself one of the elements of the partition.

Definition 1 Let $\Phi=(\mu, f)$ be a rule and let $b$ be a profile. Take $\mu(b)=\left\{S_{1}, \ldots, S_{K}\right\}=\pi$. We say that $\Phi$ is stable at $b \in[0,1]^{n}$ if there do not exist $i \in N$ and $k \in\{1, \ldots, K\}$ such that:

(1) $f_{i}\left(\pi_{-i, k}, b\right) P_{i} f_{i}(\pi, b)$, and

(2) if $S_{k} \neq S_{\pi}(i)$ then, for all $j \in S_{k}, f_{j}\left(\pi_{-i, k}, b\right) R_{j} f_{j}(\pi, b) .^{8}$

A rule $\Phi=(\mu, f)$ is stable if it is stable at all $b \in[0,1]^{n}$.

\section{$3 \quad$ Stable Rules}

In the following subsections we study the stability of rules associated to two well-known solutions: the proportional and the sequential dictator solutions.

have already said in the Introduction, we restrict our analysis to the case in which a unique solution is applied to every coalition in the partition. This requirement implies that the same principles are used across coalitions and can be interpreted as a consistency requirement.

${ }^{8}$ If there exist $i$ and $k$ such that (1) and (2) hold we say $i$ wants to leave $S_{\pi}(i)$ to join $S_{k}$ and all agents in $S_{k}$ want to admit $i$. 


\subsection{Proportional Solution}

The proportional solution $p=\left\{p^{S}\right\}_{S \subseteq N}$ is defined as follows: for each non-empty subset of agents $S \subseteq N$, each $b_{S} \in[0,1]^{s}$ and $i \in S$,

$$
p_{i}^{S}\left(b_{S}\right)= \begin{cases}\frac{b_{i}}{\sum_{j \in S} b_{j}} & \text { if } \sum_{j \in S} b_{j} \neq 0 \\ \frac{1}{\# S} & \text { otherwise }\end{cases}
$$

The proportional solution is very intuitive and has been extensively used to solve many allocation problems (for instance, in bankruptcy). If an agent's demand represents a proportion $x \%$ of the total demand in his coalition, the solution dictates that the agent should receive $x \%$ of the unit they are sharing.

Given a partition function $\mu$, define the proportional rule as $P^{\mu}=(\mu, p)$. Proposition 1 establishes that there is always a partition function such that the proportional rule is stable. The proof is constructive and proceeds by induction on the number of agents. An intuitive explanation of the structure of such partitions is as follows. We consider a non-empty set of agents $S_{1}$ such that the sum of the best shares of its agents is "closest" to 1 . By the induction hypothesis there exists a partition function $\mu^{p}\left(b_{N \backslash S_{1}}\right)$ such that the proportional rule is stable over $N \backslash S_{1}$. We take $\mu^{p}(b)=\left\{S_{1}, \mu^{p}\left(b_{N \backslash S_{1}}\right)\right\}$ and we prove that the proportional rule is stable.

Proposition 1 There exists a partition function $\mu^{p}$ such that the rule $P^{\mu^{p}}$ is stable.

Proof By induction on $n$.

- Assume $n=1$. The stability of $P^{\mu^{p}}$ is obvious.

Induction Hypothesis: For all $S$ with $1 \leq \# S<n$ there exists a partition function $\mu^{S}$ such that $\left(\mu^{S}, p=\left\{p^{T}\right\}_{T \subseteq S}\right)$ is stable.

- Consider $N$ and an arbitrary $b \in[0,1]^{n}$. Define $\mu^{p}(b)$ as follows.

First, assume $b_{i}=0$ for all $i \in N$. Then, set $\mu^{p}(b)=\{\{N\}\}$. Obviously, $\left(\mu^{p}, p\right)$ is stable at $b$.

Assume now that $b_{i}>0$ for some $i \in N$. Take an arbitrary $S_{1} \subseteq N$ with the property that

$$
S_{1} \in A M \equiv \arg \min _{\substack{S \subseteq N \\ \exists j \in S \text { s.t. } b_{j}>0}} \frac{\left|\sum_{j \in S} b_{j}-1\right|}{\sum_{j \in S} b_{j}}
$$

and $\# S_{1} \geq \# T$ for all $T \in A M$. Observe that if $b_{i}=0, i \in S_{1}$. If $S_{1}=N$, set $\mu^{p}(b)=\{\{N\}\}$. By the definition of $S_{1}, P^{\mu^{p}}(b)$ is stable at $b$. Assume now that $N \backslash S_{1} \neq \varnothing$ and consider the subprofile $b_{N \backslash S_{1}}$. Define $\mu^{p}(b)=\left\{\mu^{N \backslash S_{1}}\left(b_{N \backslash S_{1}}\right), S_{1}\right\}$. We next show that $\left(\mu^{p}(b), p(b)\right)$ is an stable allocation at $b$.

First, by definition of $S_{1}$, for all $i \in S_{1}$ and for any $T \in \mu^{N \backslash S_{1}}\left(b_{N \backslash S_{1}}\right)$,

$$
\frac{\left|\sum_{j \in S_{1}} b_{j}-1\right|}{\sum_{j \in S_{1}} b_{j}} \leq \frac{\left|\sum_{j \in T} b_{j}+b_{i}-1\right|}{\sum_{j \in T} b_{j}+b_{i}} .
$$

Then,

$$
\left|\frac{b_{i}}{\sum_{j \in S_{1}} b_{j}}-b_{i}\right|=b_{i}\left|\frac{\sum_{j \in S_{1}} b_{j}-1}{\sum_{j \in S_{1}} b_{j}}\right| \leq b_{i}\left|\frac{\sum_{j \in T} b_{j}+b_{i}-1}{\sum_{j \in T} b_{j}+b_{i}}\right|=\left|\frac{b_{i}}{\sum_{j \in T} b_{j}+b_{i}}-b_{i}\right| .
$$


Thus, $p_{i}^{S_{1}}\left(b_{S_{1}}\right) R_{i} p_{i}^{T \cup\{i\}}\left(b_{T \cup\{i\}}\right)$. Hence, $i$ does not want to leave $S_{1}$ to join $T$.

Second, take $k \in N \backslash S_{1}$. By definition of $S_{1}$,

$$
\frac{\left|\sum_{j \in S_{1}} b_{j}+b_{k}-1\right|}{\sum_{j \in S_{1}} b_{j}+b_{k}}>\frac{\left|\sum_{j \in S_{1}} b_{j}-1\right|}{\sum_{j \in S_{1}} b_{j}} .
$$

Notice that the inequality is strict since $S_{1}$ has the largest size among all sets in $A M$. Then, consider any $i \in S_{1}$ such that $b_{i}>0$. By the definition of $S_{1}$, there exists at least one agent with this property. Then,

$$
\left|\frac{b_{i}}{\sum_{j \in S_{1}} b_{j}+b_{k}}-b_{i}\right|>\left|\frac{b_{i}}{\sum_{j \in S_{1}} b_{j}}-b_{i}\right| .
$$

Thus, $p_{i}^{S_{1}}\left(b_{S_{1}}\right) P_{i} p_{i}^{S_{1} \cup\{k\}}\left(b_{S_{1} \cup\{k\}}\right)$. Hence, $i \in S_{1}$ does not want to admit $k \in N \backslash S_{1}$ in $S_{1}$.

Third, by the induction hypothesis, $\left(\mu^{N \backslash S_{1}}\left(b_{N \backslash S_{1}}\right), p^{N \backslash S_{1}}\left(b_{N \backslash S_{1}}\right)\right)$ is an stable allocation at $b_{N \backslash S_{1}}$; namely, for all $S \in \pi^{N \backslash S_{1}}\left(b_{N \backslash S_{1}}\right)$ and $k \in N \backslash S_{1}$ such that $k \notin S$, either $k$ does not want to join $S$ or there is some agent in $S$ that does not want to admit $k$.

Finally, we check that no agent in $S_{1}$ wants to leave and form a singleton coalition; namely, for all $i \in S_{1}, p_{i}^{S_{1}}\left(b_{S_{1}}\right) R_{i} p_{i}^{\{i\}}\left(b_{i}\right)$. If $b_{i}=0$, the weak preference follows immediately. Assume $b_{i}>0$. Then, the weak preference also holds because, by the definition of $S_{1}$,

$$
\left|\frac{b_{i}}{\sum_{j \in S_{1}} b_{j}}-b_{i}\right|=b_{i} \frac{\left|1-\sum_{j \in S_{1}} b_{j}\right|}{\sum_{j \in S_{1}} b_{j}} \leq b_{i} \frac{\left|1-b_{i}\right|}{b_{i}}=\left|1-b_{i}\right| .
$$

Hence, $P^{\mu^{p}}=\left(\mu^{p}, p\right)$ is an stable rule.

We finish this subsection by showing that not all weighted proportional rules are stable. To see that, let $w=\left(w_{1}, \ldots, w_{n}\right) \in(0,1)^{n}$ be a vector of weights such that $\sum_{i \in N} w_{i}=1$. The weighted proportional solution $w p=\left\{w p^{S}\right\}_{S \subseteq N}$ is defined as follows: for each non-empty subset of agents $S \subseteq N$, each $b_{S} \in[0,1]^{s}$ and $i \in S$,

$$
w p_{i}^{S}\left(b_{S}\right)= \begin{cases}\frac{w_{i} b_{i}}{\sum_{j \in S} w_{j} b_{j}} & \text { if } \sum_{j \in S} b_{j} \neq 0 \\ \frac{w_{i}}{\sum_{j \in S} w_{j}} & \text { otherwise. }\end{cases}
$$

Given a partition function $\mu$, define the weighted proportional rule as $W^{\mu}=(\mu, w p)$.

The following example shows that there are vectors of weights $w$ such that there is no partition function $\mu$ for which the weighted proportional rule $W^{\mu}=(\mu, w p)$ is stable.

Example 1 Let $N=\{1,2,3\}$ and consider the vector of weights $w=(0.4,0.2,0.4)$. Take the profile $b=(0.8,0.5,0.4)$. Then, the allocations corresponding to the five possible partitions functions are: $W^{\mu_{1}}(b)=(\{1,2,3\},(0.552,0.172,0.276)), W^{\mu_{2}}(b)=(\{\{1\},\{2,3\}\},(1,0.385,0.615))$, $W^{\mu_{3}}(b)=(\{\{1,3\},\{2\}\},(0.667,1,0.333)), W^{\mu_{4}}(b)=(\{\{1,2\},\{3\}\},(0.762,0.238,1))$ and $W^{\mu_{5}}(b)=$ $(\{\{1\},\{2\},\{3\}\},(1,1,1))$. But the strict preference $w p_{1}\left(\mu_{2}(b), b\right) P_{1} w p_{1}\left(\mu_{1}(b), b\right)$ implies that if all agents were in the same coalition, agent 1 would prefer to leave and set a new coalition by himself. Thus, $W^{\mu_{1}}$ is not an stable rule. The two strict preferences $w p_{3}\left(\mu_{3}(b), b\right) P_{3} w p_{3}\left(\mu_{2}(b), b\right)$ and $w p_{1}\left(\mu_{3}(b), b\right) P_{1} w p\left(\mu_{2}(b), b\right)$ imply that if the partition was $\{\{1\},\{2,3\}\}$ then agent 3 would rather join the coalition formed by agent 1 alone and agent 1 would be happy to admit him. Thus, $W^{\mu_{2}}$ is not an stable rule. Similarly, the three pairs (i) $w p_{1}\left(\mu_{4}(b), b\right) P_{1} w p_{1}\left(\mu_{3}(b), b\right)$ and 
$w p_{2}\left(\mu_{4}(b), b\right) P_{2} w p_{2}\left(\mu_{3}(b), b\right)$, (ii) $w p_{2}\left(\mu_{2}(b), b\right) P_{2} w p_{2}\left(\mu_{4}(b), b\right)$ and $w p_{3}\left(\mu_{2}(b), b\right) P_{3} w p_{3}\left(\mu_{4}(b), b\right)$, and (iii) $w p_{1}\left(\mu_{3}(b), b\right) P_{1} w p_{1}\left(\mu_{5}(b), b\right)$ and $w p_{3}\left(\mu_{3}(b), b\right) P_{3} w p_{3}\left(\mu_{5}(b), b\right)$ imply that $W^{\mu_{3}}, W^{\mu_{4}}$, and $W^{\mu_{5}}$ are not stable rules, respectively.

\subsection{Sequential Dictator Solutions}

Let $\sigma: N \rightarrow N$ be a one-to-one mapping defining an ordering on the set of agents $N$; namely, for $i, j \in N, \sigma(i)<\sigma(j)$ means that agent $i$ comes before agent $j$ in the ordering $\sigma$. Fix $\sigma$ and $S \neq \varnothing$. Define the sequential dictator solution associated to $\sigma$ for $S$, denoted by $\sigma d^{S}:[0,1]^{s} \rightarrow[0,1]^{s}$, as follows: for each $b_{S} \in[0,1]^{s}$ and $i \in S$,

$$
\sigma d_{i}^{S}\left(b_{S}\right)= \begin{cases}\min \left\{b_{i}, \max \left\{1-\sum_{\{j \in S \mid \sigma(j)<\sigma(i)\}} b_{j}, 0\right\}\right\} & \text { if } i \text { is s.t. } \exists j \in S, \sigma(i)<\sigma(j) \\ \max \left\{1-\sum_{\{j \in S \mid \sigma(j)<\sigma(i)\}} b_{j}, 0\right\} & \text { otherwise. }\end{cases}
$$

The sequential dictator solution associated to $\sigma$ is the family $\sigma d=\left\{\sigma d^{S}\right\}_{S \subseteq N}$, where for each non-empty subset $S \subseteq N, \sigma d^{S}$ is a sequential dictator solution associated to $\sigma$ for $S$.

An ordering on the set of agents induces an ordering on each possible coalition. Given a coalition, the sequential dictator solution uses this ordering to allocate the exact demand to the agents until the unit is exhausted or there are no more agents in the coalition. This solution has been extensively used to solve problems where a fixed set of indivisible objects have to be assigned to a set of agents with ordinal preferences on the objects (see for instance Hylland and Zeckhauser (1979)). Often, by randomizing on the set of orders ex-ante fairness can be obtained.

Given a partition function $\mu$, define the sequential dictator rule as $\sigma D^{\mu}=(\mu, \sigma d)$. Proposition 2 establishes that there is always a partition function such that the sequential dictator rule is stable. The proof is also constructive and proceeds by induction on the number of agents. An intuitive explanation of the structure of such partitions is as follows. By simplicity we take $\sigma(i)=i$ for all $i \in N$. Let $\bar{S}$ be the coalition that would result in the best allotment for agent $n$ (of course $n \in \bar{S}$ ), according with $\sigma d$ applied to $b_{\bar{S}}$. Two cases are possible: agent $n$ obtains 0 in $\bar{S}$ or agent $n$ obtains a positive share in $\bar{S}$. First case, by induction hypothesis there exists a partition function $\mu^{\sigma d}\left(b_{N \backslash\{n\}}\right)$ such that $\sigma d$ is stable over $N \backslash\{n\}$. We include $n$ in the element of $\mu^{\sigma d}\left(b_{N \backslash\{n\}}\right)$ in which agent $n$ obtains the best. With this new partition we prove that $\sigma d$ is stable over $N$. Second case, by induction hypothesis there exists a partition function $\mu^{p}\left(b_{N \backslash \bar{S}}\right)$ such that $\sigma d$ is stable over $N \backslash \bar{S}$. We take $\mu^{\sigma d}(b)=\left\{\bar{S}, \mu^{\sigma d}\left(b_{N \backslash \bar{S}}\right)\right\}$ and we prove that $\sigma d$ is stable.

Proposition 2 Let $\sigma$ be an ordering on $N$. Then, there exists a partition function $\mu^{\sigma d}$ such that the rule $\sigma D^{\mu^{\sigma d}}=\left(\mu^{\sigma d}, \sigma d\right)$ is stable.

Proof Without loss of generality we assume that $\sigma$ is such that $\sigma(i)=i$ for all $i \in N$. In the proof we will omit the reference to the ordering $\sigma$. The proof is by induction on $n$.

- Assume $n=1$. The stability of $\sigma D^{\mu^{\sigma d}}$ is obvious.

Induction Hypothesis: For all $S \subset N$ with $1 \leq \# S<n$, there exists a partition function $\mu^{d, S}$ such that $\left(\mu^{d, S}, d=\left\{d^{T}\right\}_{T \subseteq S}\right)$ is stable.

- Given $N$ and $b \in[0,1]^{n}$, select

$$
\bar{S} \in \overline{A M}=\arg \min _{\substack{S \subset N \\ n \in S}}\left|1-\sum_{i \in S} b_{i}\right|
$$


with the property that $\# \bar{S} \geq \# S$ for all $S \in \overline{A M}$. Hence, if $b_{j}=0$ then,

$$
j \in \bar{S} .
$$

Let $v=\left|1-\sum_{i \in \bar{S}} b_{i}\right|=\min _{S \subset N \backslash\{n\}}\left|1-\sum_{i \in S} b_{i}-b_{n}\right|$. In particular

$$
v \leq\left|1-b_{n}\right|
$$

We will consider the following cases:

CASE 1: $v \geq b_{n}$.

Notice that $\left|1-\sum_{i \in T} b_{i}-b_{n}\right| \geq v$ for all $T \subseteq N \backslash\{n\}$. By the induction hypothesis the allocation $\left(\mu^{d, N \backslash\{n\}}\left(b_{N \backslash\{n\}}\right), d^{N \backslash\{n\}}\left(b_{N \backslash\{n\}}\right)\right)$ is stable. Let $\hat{S}$ be such that $\hat{S} \in \mu^{d, N \backslash\{n\}}\left(b_{N \backslash\{n\}}\right)$ and, for all $S \in \mu^{d, N \backslash\{n\}}\left(b_{N \backslash\{n\}}\right), \sum_{i \in S} b_{i} \leq \sum_{i \in \hat{S}} b_{i}$. Define $\mu^{d, N}(b)=\left\{\left\{\mu^{d, N \backslash\{n\}}\left(b_{N \backslash\{n\}}\right) \backslash \hat{S}\right\}, \hat{S} \cup\{n\}\right\}$. We want to show that $\left(\mu^{d, N}(b), d^{N}(b)\right)$ is stable at $b$.

Assume first that $\hat{S} \cup\{n\}=N$. Observe that

$$
d_{n}^{N}(b)= \begin{cases}0 & \text { if } \sum_{i \in N \backslash\{n\}} b_{i} \geq 1 \\ 1-\sum_{i \in N \backslash\{n\}} b_{i} & \text { otherwise. }\end{cases}
$$

Since $v \geq b_{n}$, for all $j<n, d_{j}^{N \backslash\{n\}}\left(b_{N \backslash\{n\}}\right)=d_{j}^{N}(b)$. By the induction hypothesis, $\left|d_{j}^{N \backslash\{n\}}\left(b_{N \backslash\{n\}}\right)-b_{j}\right| \leq$ $\left|1-b_{j}\right|$. Thus, $\left|d_{j}^{N}(b)-b_{j}\right| \leq\left|1-b_{j}\right|$; namely, $j$ does not want to leave the set $\hat{S} \cup\{n\}=N$.

We will show that $n$ does not want to leave $N$ either. Assume the contrary,

$$
\left|1-b_{n}\right|<\left|d_{n}^{N}(b)-b_{n}\right|
$$

If $d_{n}^{N}(b)=0$ then, $\left|1-b_{n}\right|<\left|0-b_{n}\right| ;$ i.e., $b_{n}>\frac{1}{2}$. By the hypothesis of Case 1 and $(2), \frac{1}{2}<$ $b_{n} \leq v \leq 1-b_{n}$, a contradiction. If $d_{n}^{N}(b)=1-\sum_{i \in N \backslash\{n\}} b_{i}>0$ then, by $(3), b_{n} \leq v \leq$ $\left|1-b_{n}\right|<\left|1-\sum_{i \in N \backslash\{n\}} b_{i}-b_{n}\right|$. If $1-\sum_{i \in N \backslash\{n\}} b_{i}-b_{n}>0$ then, $1-b_{n}<1-\sum_{i \in N \backslash\{n\}} b_{i}-b_{n}$, a contradiction. If $1-\sum_{i \in N \backslash\{n\}} b_{i}-b_{n}<0$ then, by the alternative definition of $v$ as the $\min _{S \subset N \backslash\{n\}}\left|1-\sum_{i \in S} b_{i}-b_{n}\right|$ and the fact that $v \geq b_{n}, b_{n} \leq \sum_{i \in N \backslash\{n\}} b_{i}+b_{n}-1$. Hence, $1-$ $\sum_{i \in N \backslash\{n\}} b_{i} \leq 0$, a contradiction. Thus, $\left(\{\{N\}\}, d^{N}(b)\right)$ is an stable allocation at $b$.

Assume now that $N \backslash(\hat{S} \cup\{n\}) \neq \varnothing$. We distinguish between the following two subcases.

SubCase 1.1: $\sum_{i \in \hat{S}} b_{i} \geq 1$.

Then, $d_{n}^{\hat{S} \cup\{n\}}\left(b_{\hat{S} \cup\{n\}}\right)=0$ and for all $i \in \hat{S}$,

$$
d_{i}^{\hat{S} \cup\{n\}}\left(b_{\hat{S} \cup\{n\}}\right)=d_{i}^{\hat{S}}\left(b_{\hat{S}}\right) .
$$

First, by (4) and the induction hypothesis, no agent in $\hat{S}$ wants to leave $\hat{S}$. Moreover, by the hypothesis of Case 1 and (2), $b_{n} \leq \frac{1}{2}$ and hence, $n$ does not want to leave $\hat{S} \cup\{n\}$ and to form a singleton coalition.

Second, take any $j \in N \backslash(\hat{S} \cup\{n\})$. Observe that $j<n$. Then, since $\sum_{i \in \hat{S}} b_{i} \geq 1$,

$$
d_{j}^{\hat{S} \cup\{j\} \cup\{n\}}\left(b_{\hat{S} \cup\{j\} \cup\{n\}}\right)=d_{j}^{\hat{S} \cup\{j\}}\left(b_{\hat{S} \cup\{j\}}\right) .
$$


By the induction hypothesis, the allocation $\left(\mu^{d, N \backslash\{n\}}\left(b_{N \backslash\{n\}}\right), d^{N \backslash\{n\}}\left(b_{N \backslash\{n\}}\right)\right)$ is stable. Define $\pi^{N \backslash\{n\}}=\mu^{d, N \backslash\{n\}}\left(b_{N \backslash\{n\}}\right)$. Hence, and since $\hat{S} \in \pi^{N \backslash\{n\}}$ and $j \notin \hat{S}$, either there exists $i \in \hat{S}$ such that

$$
d_{i}^{\hat{S}}\left(b_{\hat{S}}\right) P_{i} d_{i}^{\hat{S} \cup\{j\}}\left(b_{\hat{S} \cup\{j\}}\right)
$$

or else

$$
d_{j}^{S_{\pi^{N} \backslash\{n\}}(j)}\left(b_{S_{\pi^{N} \backslash\{n\}}(j)}\right) R_{j} d_{j}^{\hat{S} \cup\{j\}}\left(b_{\hat{S} \cup\{j\}}\right) .
$$

Thus, by (4) and (5), either

$$
d_{i}^{\hat{S} \cup\{n\}}\left(b_{\hat{S} \cup\{n\}}\right) P_{i} d_{i}^{\hat{S} \cup\{j\} \cup\{n\}}\left(b_{\hat{S} \cup\{j\} \cup\{n\}}\right)
$$

or else

$$
d_{j}^{S \pi^{N \backslash\{n\}}(j)}\left(b_{S_{\pi^{N} \backslash\{n\}}(j)}\right) R_{j} d_{j}^{\hat{S} \cup\{j\} \cup\{n\}}\left(b_{\hat{S} \cup\{j\} \cup\{n\}}\right) .
$$

Namely, either $j$ is not admitted in $\hat{S} \cup\{n\}$ or else $j$ does not want to leave $S_{\pi^{N \backslash\{n\}}}(j)$ to join $\hat{S} \cup\{n\}$.

Third, take any $T \in \mu^{d, N}(b) \backslash(\hat{S} \cup\{n\})$ and consider the coalition $T \cup\{n\}$. If $\sum_{i \in T} b_{i} \geq 1$ then $d_{n}^{T \cup\{n\}}\left(b_{T \cup\{n\}}\right)=d_{n}^{\hat{S} \cup\{n\}}\left(b_{\hat{S} \cup\{n\}}\right)=0$ and $n$ does not want to leave $\hat{S} \cup\{n\}$ to join $T$. If $\sum_{i \in T} b_{i}<1$ then, $d_{n}^{T \cup\{n\}}\left(b_{T \cup\{n\}}\right)=1-\sum_{i \in T} b_{i}$. Since $\left|d_{n}^{T \cup\{n\}}\left(b_{T \cup\{n\}}\right)-b_{n}\right|=\left|1-\sum_{i \in T} b_{i}-b_{n}\right| \geq v \geq b_{n}=$ $\left|d_{n}^{\hat{S} \cup\{n\}}\left(b_{\hat{S} \cup\{n\}}\right)-b_{n}\right|, d_{n}^{\hat{S} \cup\{n\}}\left(b_{\hat{S} \cup\{n\}}\right) R_{n} d_{n}^{T \cup\{n\}}\left(b_{T \cup\{n\}}\right)$. Thus, $n$ does not want to leave $\hat{S} \cup\{n\}$ to join $T$.

SubCASE 1.2: $\sum_{i \in \hat{S}} b_{i}<1$.

Notice that, by the definition of $\hat{S}$, for all $S \in \pi^{N \backslash\{n\}}$,

$$
\sum_{i \in S} b_{i}<1
$$

First, take $j \in S^{\prime} \in \mu^{d, N}(b) \backslash(\hat{S} \cup\{n\})$. Using the fact that, by the induction hypothesis, $j$ and $\hat{S}$ did not generate an instability in the allocation $\left(\pi^{N \backslash\{n\}}, d^{N \backslash\{n\}}\left(b_{N \backslash\{n\}}\right)\right)$ we will show that $j$ and $\hat{S} \cup\{n\}$ do not generate an instability in the allocation $\left(\mu^{d, N}(b), d^{N}(b)\right)$. Assume

$$
d_{j}^{S^{\prime}}\left(b_{S^{\prime}}\right) \neq b_{j}
$$

otherwise, $j$ does not want to leave $S^{\prime}$. By (6), $\sum_{i \in S^{\prime}} b_{i}<1$. Hence, $j=\max _{i \in S^{\prime}} i$ and $d_{j}^{S^{\prime}}\left(b_{S^{\prime}}\right)=$ $1-\sum_{i \in S^{\prime} \backslash\{j\}} b_{i}>b_{j}$.

Assume $\sum_{i \in \hat{S}} b_{i}+b_{j} \geq 1$. Then, since $\sum_{i \in \hat{S}} b_{i}<1$ and $i<n$ for all $i \in \hat{S}$,

$$
d_{i}^{\hat{S} \cup\{j\}}\left(b_{\hat{S} \cup\{j\}}\right)=d_{i}^{\hat{S} \cup\{j\} \cup\{n\}}\left(b_{\hat{S} \cup\{j\} \cup\{n\}}\right) .
$$

By the stability of the allocation $\left(\pi^{N \backslash\{n\}}, d^{N \backslash\{n\}}\left(b_{N \backslash\{n\}}\right)\right)$ either

$$
\left|d_{j}^{S^{\prime}}\left(b_{S^{\prime}}\right)-b_{j}\right| \leq\left|d_{j}^{\hat{S} \cup\{j\}}\left(b_{\hat{S} \cup\{j\}}\right)-b_{j}\right|
$$

or else there must exist $i^{\prime} \in \hat{S}$ such that

$$
\left|d_{i^{\prime}}^{\hat{S}}\left(b_{\hat{S}}\right)-b_{i^{\prime}}\right|<\left|d_{i^{\prime}}^{\hat{S} \cup\{j\}}\left(b_{\hat{S} \cup\{j\}}\right)-b_{i^{\prime}}\right| .
$$


If (9) holds then, by (8),

$$
\left|d_{j}^{S^{\prime}}\left(b_{S^{\prime}}\right)-b_{j}\right| \leq\left|d_{j}^{\hat{S} \cup\{j\} \cup\{n\}}\left(b_{\hat{S} \cup\{j\} \cup\{n\}}\right)-b_{j}\right|,
$$

namely, $j$ does not want to leave $S^{\prime}$ to join $\hat{S} \cup\{n\}$. Assume (9) does not hold; i.e. $j$ wants lo leave $S^{\prime}$ to join $\hat{S}$. Then, (10) holds. Assume that $j$ wants to leave $S^{\prime}$ to join $\hat{S} \cup\{n\}$; that is,

$$
\left|d_{j}^{S^{\prime}}\left(b_{S^{\prime}}\right)-b_{j}\right|>\left|d_{j}^{\hat{S} \cup\{j\} \cup\{n\}}\left(b_{\hat{S} \cup\{j\} \cup\{n\}}\right)-b_{j}\right| .
$$

Then, by (10), (8) and $d_{i^{\prime}}^{\hat{S} \cup\{n\}}\left(b_{\hat{S} \cup\{n\}}\right)=b_{i^{\prime}}$,

$$
\begin{aligned}
\left|d_{i^{\prime}}^{\hat{S} \cup\{n\}}\left(b_{\hat{S} \cup\{n\}}\right)-b_{i^{\prime}}\right| & =0 \leq\left|d_{i^{\prime}}^{\hat{S}}\left(b_{\hat{S}}\right)-b_{i^{\prime}}\right| \\
& <\left|d_{i^{\prime}}^{\hat{S} \cup\{j\}}\left(b_{\hat{S} \cup\{j\}}\right)-b_{i^{\prime}}\right| \\
& =\left|d_{i^{\prime}}^{\hat{S} \cup\{j\} \cup\{n\}}\left(b_{\hat{S} \cup\{j\} \cup\{n\}}\right)-b_{i^{\prime}}\right| .
\end{aligned}
$$

Thus, $i^{\prime}$ does not want to admit $j$ in the coalition $\hat{S} \cup\{n\}$.

Assume $\sum_{i \in \hat{S}} b_{i}+b_{j}<1$. Then,

$$
d_{j}^{\hat{S} \cup\{j\}}\left(b_{\hat{S} \cup\{j\}}\right)= \begin{cases}b_{j} & \text { if } j \text { is not the last in } \hat{S} \cup\{j\} \\ 1-\sum_{i \in \hat{S}} b_{i} & \text { if } j \text { is the last in } \hat{S} \cup\{j\} .\end{cases}
$$

Assume first that $j$ is not the last in $\hat{S} \cup\{j\}$. Then, $d_{j}^{\hat{S} \cup\{j\}}\left(b_{\hat{S} \cup\{j\}}\right)=b_{j}$. By $(7)$,

$$
d_{j}^{\hat{S} \cup\{j\}}\left(b_{\hat{S} \cup\{j\}}\right) P_{j} d_{j}^{S^{\prime}}\left(b_{S^{\prime}}\right) .
$$

Let $j^{*}=\max _{i \in \hat{S}} i>j$. For all $i \in \hat{S} \backslash\left\{j^{*}\right\}$,

$$
d_{i}^{\hat{S} \cup\{j\}}\left(b_{\hat{S} \cup\{j\}}\right)=d_{i}^{\hat{S}}\left(b_{\hat{S}}\right) .
$$

Moreover, since $d_{j^{*}}^{\hat{S}}\left(b_{\hat{S}}\right)=1-\sum_{i \in \hat{S}} b_{i}+b_{j^{*}}$ and $d_{j^{*}}^{\hat{S} \cup\{j\}}\left(b_{\hat{S} \cup\{j\}}\right)=1-\sum_{i \in \hat{S}} b_{i}+b_{j^{*}}-b_{j}$,

$$
\left|d_{j^{*}}^{\hat{S}}\left(b_{\hat{S}}\right)-b_{j^{*}}\right|=\left|1-\sum_{i \in \hat{S}} b_{i}\right| \geq\left|1-\sum_{i \in \hat{S}} b_{i}-b_{j}\right|=\left|d_{j^{*}}^{\hat{S} \cup j j\}}\left(b_{\hat{S} \cup\{j\}}\right)-b_{j^{*}}\right| .
$$

Hence,

$$
d_{j^{*}}^{\hat{S} \cup\{j\}}\left(b_{\hat{S} \cup\{j\}}\right) R_{j^{*}} d_{j^{*}}^{\hat{S}}\left(b_{\hat{S}}\right) .
$$

Conditions (12), (13), and (14) imply that $j$ wants to leave $S^{\prime}$ to join $\hat{S}$ and all agents in $\hat{S}$ want to admit $j$, contradicting that $\left(\pi^{N \backslash\{n\}}, d^{N \backslash\{n\}}\left(b_{N \backslash\{n\}}\right)\right)$ is an stable allocation.

Assume now that $j$ is the last in $\hat{S} \cup\{j\}$. Hence, for all $i \in \hat{S}, d_{i}^{\hat{S} \cup\{j\}}\left(b_{\hat{S} \cup\{j\}}\right) R_{i} d_{i}^{\hat{S}}\left(b_{\hat{S}}\right)$. Moreover, since $\sum_{i \in S^{\prime}} b_{i} \leq \sum_{i \in \hat{S}} b_{i}$ and $\sum_{i \in \hat{S}} b_{i}+b_{j}<1$, if either $b_{j} \neq 0$ or $\sum_{i \in S^{\prime}} b_{i}<\sum_{i \in \hat{S}} b_{i}$ then,

$$
1-\sum_{i \in S^{\prime}} b_{i}+b_{j}>1-\sum_{i \in \hat{S}} b_{i}>b_{j} .
$$

Since $d_{j}^{S^{\prime}}\left(b_{S^{\prime}}\right)=1-\sum_{i \in S^{\prime}} b_{i}+b_{j}$ and $d_{j}^{\hat{S} \cup\{j\}}\left(b_{\hat{S} \cup\{j\}}\right)=1-\sum_{i \in \hat{S}} b_{i}, j$ wants to leave $S^{\prime}$ to join $\hat{S}$ and all agents in $\hat{S}$ want to admit $j$, contradicting that $\left(\pi^{N \backslash\{n\}}, d^{N \backslash\{n\}}\left(b_{N \backslash\{n\}}\right)\right)$ is an stable 
allocation. Assume now that $b_{j}=0$ and $\sum_{i \in S^{\prime}} b_{i}=\sum_{i \in \hat{S}} b_{i}$. We can prove that $j^{*}$ wants to leave $\hat{S}$ to join $S^{\prime}$ and no agent of $S^{\prime}$ rejects $j^{*}$. If $b_{j^{*}}=0$ then, we get a contradiction of the stability of $\left(\pi^{N \backslash\{n\}}, d^{N \backslash\{n\}}\left(b_{N \backslash\{n\}}\right)\right)$ proceeding as above. If $b_{j^{*}}>0$ then, changing the roles of $\hat{S}$ and $S^{\prime}$ and $j^{*}$ and $j$ we will get a contradiction of the stability of $\left(\pi^{N \backslash\{n\}}, d^{N \backslash\{n\}}\left(b_{N \backslash\{n\}}\right)\right)$. Observe that this can be done since $b_{j}=0$ and $\sum_{i \in S^{\prime}} b_{i}=\sum_{i \in \hat{S}} b_{i}$.

Second, take any $T \in \mu^{d, N}(b) \backslash(\hat{S} \cup\{n\})$. We want to check that $n$ does not want to leave $\hat{S} \cup\{n\}$ to join $T$. Since, by definition of $\hat{S}, \sum_{i \in T} b_{i} \leq \sum_{i \in \hat{S}} b_{i}$,

$$
d_{n}^{T \cup\{n\}}\left(b_{T \cup\{n\}}\right)=1-\sum_{i \in T} b_{i} \geq 1-\sum_{i \in \hat{S}} b_{i}=d_{n}^{\hat{S} \cup\{n\}}\left(b_{\hat{S} \cup\{n\}}\right)>b_{n},
$$

where the strict inequality follows because otherwise, if $d_{n}^{\hat{S} \cup\{n\}}\left(b_{\hat{S} \cup\{n\}}\right) \leq b_{n}$ then, $1-\sum_{i \in \hat{S}} b_{i}-$ $b_{n} \leq 0$, implying that

$$
b_{n} \leq v=\left|1-\sum_{i \in \bar{S}} b_{i}\right| \leq\left|1-\sum_{i \in \hat{S}} b_{i}-b_{n}\right|=\sum_{i \in \hat{S}} b_{i}+b_{n}-1,
$$

a contradiction with the hypothesis of Subcase 1.2 stating that $\sum_{i \in \hat{S}} b_{i}<1$. Thus, by singlepeakedness, $d_{n}^{\hat{S} \cup\{n\}}\left(b_{\hat{S} \cup\{n\}}\right) R_{n} d_{n}^{T \cup\{n\}}\left(b_{T \cup\{n\}}\right)$. Hence, $n$ does not want to leave $\hat{S} \cup\{n\}$ to join coalition $T$.

Third, we show that $n$ does not want to leave $\hat{S} \cup\{n\}$ to form a singleton coalition. Assume otherwise; i.e.,

$$
\left|1-b_{n}\right|<\left|1-\sum_{i \in \hat{S}} b_{i}-b_{n}\right|
$$

holds. If $1-\sum_{i \in \hat{S}} b_{i}-b_{n}>0$ then, (15) implies that $\sum_{i \in \hat{S}} b_{i}<0$, a contradiction. If $1-\sum_{i \in \hat{S}} b_{i}-$ $b_{n} \leq 0$ then, and since $b_{n} \leq v \leq\left|1-b_{n}\right|$, (15) implies that $b_{n} \leq \sum_{i \in \hat{S}} b_{i}+b_{n}-1$, a contradiction with $\sum_{i \in \hat{S}} b_{i}<1$.

CASE 2: $v<b_{n}$.

Recall that $v=\left|1-\sum_{i \in \bar{S}} b_{i}\right|$ where $\bar{S} \in \overline{A M}=\arg \min _{\substack{S \subset N \\ n \in S}}\left|1-\sum_{i \in S} b_{i}\right|$ with the property that $\# \bar{S} \geq \# S$ for all $S \in \overline{A M}$. Observe that

$$
\sum_{i \in \bar{S} \backslash\{n\}} b_{i}<1
$$

otherwise, if $\sum_{i \in \bar{S} \backslash\{n\}} b_{i} \geq 1$ then, $v=\sum_{i \in \bar{S} \backslash\{n\}} b_{i}+b_{n}-1$. Hence, $v-b_{n}=\sum_{i \in \bar{S} \backslash\{n\}} b_{i}-1 \geq 0$, which contradicts the assumption that $v<b_{n}$.

First, assume that $\bar{S}=N$. Define $\mu^{d}(b)=\{\{N\}\}$. To obtain a contradiction, suppose that $\left(\{\{N\}\}, d^{N}(b)\right)$ is not an stable allocation. By (16), for all $i \neq n, d_{i}^{N}(b)=b_{i}$. Hence, it has to be agent $n$ who wants to leave $N$ to form a singleton coalition; that is, $d_{n}^{\{n\}}\left(b_{n}\right) P_{n} d_{n}^{N}(b)$, or equivalently, $\left|1-b_{n}\right|<\left|1-\sum_{i \in \bar{S} \backslash\{n\}} b_{i}-b_{n}\right|=v$, a contradiction with (2).

Thus assume that $\bar{S} \subsetneq N$. By the induction hypothesis, $\left(\mu^{d, N \backslash \bar{S}}\left(b_{N \backslash \bar{S}}\right), d^{N \backslash \bar{S}}\left(b_{N \backslash \bar{S}}\right)\right)$ is an stable allocation. Define $\mu^{d, N}(b)=\mu^{d, N \backslash \bar{S}}\left(b_{N \backslash \bar{S}}\right) \cup \bar{S} \equiv \pi^{N}$. To prove that the allocation $\left(\pi^{N}, d^{N}(b)\right)$ is stable, we first check that $n$ does not want to leave $\bar{S}$ to form a singleton coalition. Assume otherwise; then, by (16), $\left|1-b_{n}\right|<\left|1-\sum_{i \in \bar{S} \backslash\{n\}} b_{i}-b_{n}\right|=v$, a contradiction with (2). 
We now distinguish between the following two subcases.

SubCase 2.1: $\sum_{i \in \bar{S}} b_{i}>1$.

By $(16), d_{n}^{\bar{S}}\left(b_{\bar{S}}\right)=b_{n}-v>0$.

First, take $j \in N \backslash \bar{S}$ and consider the coalition $\bar{S} \cup\{j\}$. Then, $d_{n}^{\bar{S} \cup\{j\}}\left(b_{\bar{S} \cup\{j\}}\right)=\max \{1-$ $\left.\sum_{i \in \bar{S} \backslash\{n\}} b_{i}-b_{j}, 0\right\}<b_{n}-v=d_{n}^{\bar{S}}\left(b_{\bar{S}}\right)<b_{n}$, where the first strict inequality holds because by (1), $j \notin \bar{S}$ implies $b_{j}>0$ and $v=\sum_{i \in \bar{S}} b_{i}-1$ implies that $1-\sum_{i \in \bar{S} \backslash\{n\}} b_{i}=b_{n}-v$. Thus, by symmetric single-peakedness, $d_{n}^{\bar{S}}\left(b_{\bar{S}}\right) P_{n} d_{n}^{\bar{S} \cup\{j\}}\left(b_{\bar{S} \cup\{j\}}\right)$. Hence, $n$ does not want to admit $j$ in $\bar{S}$.

Second, by $(16), d_{i}^{\bar{S}}\left(b_{\bar{S}}\right)=b_{i}$, for all $i \in \bar{S} \backslash\{n\}$. Hence, no agent in $\bar{S} \backslash\{n\}$ wants to leave $\bar{S}$ to join any other coalition.

Third, let $T \in \mu^{d, N \backslash \bar{S}}\left(b_{N \backslash \bar{S}}\right)$ and consider the coalition $T \cup\{n\}$. Then, $d_{n}^{T \cup\{n\}}\left(b_{T \cup\{n\}}\right)=$ $\max \left\{1-\sum_{i \in T} b_{i}, 0\right\}$. If $1-\sum_{i \in T} b_{i} \geq 0$ then, by the definition of $\bar{S},\left|d_{n}^{T \cup\{n\}}\left(b_{T \cup\{n\}}\right)-b_{n}\right| \geq$ $\left|1-\sum_{i \in \bar{S} \backslash\{n\}} b_{i}-b_{n}\right|=v=\left|d_{n}^{\bar{S}}\left(b_{\bar{S}}\right)-b_{n}\right|$, and if $1-\sum_{i \in T} b_{i}<0$, then $\left|0-b_{n}\right|=b_{n}>v=$ $\left|d_{n}^{\bar{S}}\left(b_{\bar{S}}\right)-b_{n}\right|$. In the two cases we have that $d_{n}^{\bar{S}}\left(b_{\bar{S}}\right) R_{n} d_{n}^{T \cup\{n\}}\left(b_{T \cup\{n\}}\right)$. Hence, $n$ does not want to leave $\bar{S}$ to join $T$.

SUBCASE 2.2: $\sum_{i \in \bar{S}} b_{i}<1$.

By definition of $\bar{S}, d_{n}^{\bar{S}}\left(b_{\bar{S}}\right)=b_{n}+v=1-\sum_{i \in \bar{S} \backslash\{n\}} b_{i}>b_{n}$.

First, take $j \in N \backslash \bar{S}$ and consider the coalition $\bar{S} \cup\{j\}$. Then, $d_{n}^{\bar{S} \cup\{j\}}\left(b_{\bar{S} \cup\{j\}}\right)=\max \{1-$ $\left.\sum_{i \in \bar{S} \backslash\{n\}} b_{i}-b_{j}, 0\right\}$. If $d_{n}^{\bar{S} \cup\{j\}}\left(b_{\bar{S} \cup\{j\}}\right)=0$ then, $\left|d_{n}^{\bar{S} \cup\{j\}}\left(b_{\bar{S} \cup\{j\}}\right)-b_{n}\right|=b_{n}>v=\left|d_{n}^{\bar{S}}\left(b_{\bar{S}}\right)-b_{n}\right|$. If $d_{n}^{\bar{S} \cup\{j\}}\left(b_{\bar{S} \cup\{j\}}\right)=1-\sum_{i \in \bar{S} \backslash\{n\}} b_{i}-b_{j}>0$ then, $\left|d_{n}^{\bar{S} \cup\{j\}}\left(b_{\bar{S} \cup\{j\}}\right)-b_{n}\right|=\left|1-\sum_{i \in \bar{S} \cup\{j\}} b_{i}\right|>$ $v=\left|d_{n}^{\bar{S}}\left(b_{\bar{S}}\right)-b_{n}\right|$, where the strict inequality holds because, by (1), $j \notin \bar{S}$ implies $b_{j}>0$. Thus, in both cases $d_{n}^{\bar{S}}\left(b_{\bar{S}}\right) P_{n} d_{n}^{\bar{S} \cup\{j\}}\left(b_{\bar{S}\{j\}}\right)$. Hence, $n$ does not want to admit $j$ in $\bar{S}$.

Second, and since $d_{i}^{\bar{S}}\left(b_{\bar{S}}\right)=b_{i}$ for all $i \in \bar{S} \backslash\{n\}$, no agent in $\bar{S} \backslash\{n\}$ wants to leave $\bar{S}$ to join any other coalition.

Third, let $T \in \mu^{d, N \backslash \bar{S}}\left(b_{N \backslash \bar{S}}\right)$ and consider the coalition $T \cup\{n\}$. Then, $d_{n}^{T \cup\{n\}}\left(b_{T \cup\{n\}}\right)=$ $\max \left\{1-\sum_{i \in T} b_{i}, 0\right\}$. If $1-\sum_{i \in T} b_{i}>0$, and since $\left|1-\sum_{i \in T} b_{i}-b_{n}\right| \geq v$ by definition of $\bar{S}$, we have that $\left|1-\sum_{i \in T} b_{i}-b_{n}\right| \geq v=\left|d_{n}^{\bar{S}}\left(b_{\bar{S}}\right)-b_{n}\right|$. If $1-\sum_{i \in T} b_{i} \leq 0$ then, $\left|0-b_{n}\right|=b_{n}>v=$ $\left|d_{n}^{\bar{S}}\left(b_{\bar{S}}\right)-b_{n}\right|$. Thus, in both situations $\left|d_{n}^{T \cup\{n\}}\left(b_{T \cup\{n\}}\right)-b_{n}\right| \geq\left|d_{n}^{\bar{S}}\left(b_{\bar{S}}\right)-b_{n}\right|$, which implies that $d_{n}^{\bar{S}}\left(b_{\bar{S}}\right) R_{n} d_{n}^{T \cup\{n\}}\left(b_{T \cup\{n\}}\right)$. Hence, $n$ does not want to leave $\bar{S}$ to join $T$.

\subsection{Incompatibilities}

In this subsection we show that stability is incompatible with other desirable properties such as strategy-proofness, efficiency, anonymity, and envy-freeness.

Before proceeding with the formal definition of these properties a comment about these incompatibilities is in order. Since the proportional solution is not strategy-proof and the serial dictatorship solution is not anonymous, it is natural that pairing them with a stable partition function preserves these negative features. However, the reader may be surprised by the fact that the other incompatibilities also hold; for instance, that anonymity can not be satisfied after pairing the proportional solution with a stable partition function. But after all, this is not that surprising since, as the examples at the end of Remarks 1 and 2 below show, stability at a particular profile 
with two identical agents might require that they belong to two different coalitions and hence, they might receive different shares.

Strategy-proofness says that no agent obtains a better share by misreporting his best share.

Definition 2 A rule $\Phi=(\mu, f)$ is manipulable at $b \in[0,1]^{n}$ if there exist $i \in N$ and $b_{i}^{\prime} \in[0,1]$ such that

$$
f_{i}\left(\mu\left(b_{i}^{\prime}, b_{-i}\right),\left(b_{i}^{\prime}, b_{-i}\right)\right) P_{i} f_{i}\left(\mu\left(b_{i}, b_{-i}\right),\left(b_{i}, b_{-i}\right)\right) .
$$

A rule $\Phi=(\mu, f)$ is strategy-proof if it is not manipulable at any $b \in[0,1]^{n}$.

Efficiency says that the rule always selects efficient allocations.

Definition 3 An allocation $(\pi, x) \in A$ is efficient at profile $b \in[0,1]^{n}$ if it does not have a Pareto improvement; that is, there does not exist another allocation $(\gamma, y) \in A$ such that $y_{i} R_{i} x_{i}$ for all $i \in N$ and $y_{j} P_{j} x_{j}$ for at least one $j \in N$.

A rule $\Phi=(\mu, f)$ is efficient if for all $b \in[0,1]^{n}, \Phi(b)$ is an efficient allocation at $b$.

Anonymity says that the name of the agents should not matter. Let $o: N \rightarrow N$ be a one-to-one mapping and let $b \in[0,1]^{n}$ be a profile. Define the profile $b^{o}=\left(b_{o(1)}, \ldots, b_{o(n)}\right) \in[0,1]^{n}$.

Definition 4 A rule $\Phi=(\mu, f)$ is anonymous if for all one-to-one mapping $o: N \rightarrow N$ and all $b \in[0,1]^{n}, f_{i}(\mu(b), b)=f_{o(i)}\left(\mu\left(b^{o}\right), b^{o}\right)$ for all $i \in N$.

Envy-freeness says that no agent strictly prefers the share of another agent.

Definition 5 A rule $\Phi=(\mu, f)$ is envy-free if for all $b \in[0,1]^{n}$ and all $i, j \in N, f_{i}(\mu(b), b) R_{i} f_{j}(\mu(b), b)$.

Remark 1 There is no partition function $\mu$ for which the proportional rule $(\mu, p)$ is stable and satisfies one of the following properties: strategy-proofness, efficiency, anonymity, and envy-freeness.

To see that stability and strategy-proofness are incompatible, let $N=\{1,2\}$ and consider the profile $b=(0.5,0.4)$. The only stable allocation is $\left(\mu^{p}(b), p\left(\mu^{p}(b), b\right)\right)=(\{\{1,2\}\},(0.56,0.44))$. Let $b_{2}^{\prime}=0,33$. Then, $\mu^{p}\left(b_{1}, b_{2}^{\prime}\right)=\{\{1,2\}\}$ and $p\left(\mu^{p}\left(b_{1}, b_{2}^{\prime}\right),\left(b_{1}, b_{2}^{\prime}\right)\right)=(0.6,0.4)$. Thus, $0.4=$ $p_{2}\left(\mu^{p}\left(b_{1}, b_{2}^{\prime}\right),\left(b_{1}, b_{2}^{\prime}\right)\right) P_{2} p_{2}\left(\mu^{p}(b), b\right)=0.44$ and hence $\left(\mu^{p}, p\right)$ is not strategy-proof.

To see that stability and efficiency are incompatible, let $N=\{1,2\}$ and consider the profile $b=(0.4,0.9)$. Now, the only stable allocation is $\left(\mu^{p}(b), p\left(\mu^{p}(b), b\right)\right)=(\{\{1\},\{2\}\},(1,1))$. However, the allocation $(\{\{1,2\}\},(0.1,0.9))$ is a Pareto improvement.

To see that stability and anonymity or envy-freeness are incompatible, let $N=\{1,2,3\}$ and consider the profile $b=(0.3,0.3,0.8)$. It is easy to see that the only stable allocations are $\left(\{\{1\},\{2,3\}\},\left(1, \frac{0.3}{1.1}, \frac{0.8}{1.1}\right)\right)$ and $\left(\{\{2\},\{1,3\}\},\left(\frac{0.3}{1.1}, 1, \frac{0.8}{1.1}\right)\right)$. Agent 1 and agent 2 have the same best shares but in both allocations they receive a different share and either agent 1 envies agent 2 (first allocation) or the opposite (second allocation).

Remark 2 Fix an ordering $\sigma$ on $N$. There is no partition function $\mu$ for which the sequential dictator rule $(\mu, \sigma d)$ is stable and satisfies one of the following properties: strategy-proofness, efficiency, anonymity, and envy-freeness.

To see that stability and strategy-proofness are incompatible, let $N=\{1,2,3\}$ and consider $\sigma(i)=i$ for each $i=1,2,3$, and the profile $b=(0.6,0.5,0.6)$. The only stable allocation at $b$ is $\left(\mu^{\sigma d}(b), \sigma d(b)\right)=(\{\{1\},\{2,3\}\},(1,0.5,0.5))$. Take $b_{1}^{\prime}=0.4$. Since the only stable allocation at 
$b^{\prime}=\left(b_{1}^{\prime}, b_{2}, b_{3}\right)$ is $\left(\mu^{\sigma d}\left(b^{\prime}\right), \sigma d\left(b^{\prime}\right)\right)=(\{\{1,3\},\{2\}\},(0.4,1,0.6))$ and $0.4 P_{1} 1$, we conclude that for every $\mu$ for which $(\mu, \sigma d)$ is stable, $(\mu, \sigma d)$ is not strategy-proof.

Moreover, stability and efficiency are incompatible. To see it, let $N=\{1,2\}$ and consider $\sigma(1)=1, \sigma(2)=2$, and the profile $b=(0.5,0.8)$. The only stable allocation at $b$ is $\left(\mu^{\sigma d}(b), \sigma d(b)\right)=$ $(\{\{1\},\{2\}\},(1,1))$, but the allocation $(\{\{1,2\}\},(0.3,0.7))$ is a Pareto improvement.

To see that stability and anonymity or envy-freeness are incompatible, let $N=\{1,2\}$ and consider $\sigma(1)=1, \sigma(2)=2$, and the profile $b=(0.6,0.6)$. Then, the allocation $(\{1,2\},(0.6,0.4))$ is stable but the two agents have the same best shares, receive a different share and agent 2 envies agent 1 . Finally, the allocation $(\{\{1\},\{2\}\},(1,1))$ is not stable since 1 wants to join $\{2\}$ and 2 wants to admit 1 .

\section{Final Comments}

We finish the paper with three comments. First we discuss other widely used solutions: the uniform solution and the equal gain-losses. Second, we provide a positive result on the existence of efficient rules and finally, we discuss the assumption of symmetry of preferences.

\subsection{Uniform and Equal Gains-losses Solutions}

Although we do not have a proof for the existence of stable rules associated to the uniform and equal gains-losses solutions, we conjecture, based on simulations, that there exist such stable rules. Below we provide an algorithm that computes the stable partition given a vector of best shares for both the uniform and the equal gains-losses solutions. The algorithm is not proven to converge, and hence cannot be used as a proof of existence; however, we have never found a vector of best shares for which it does not converge. ${ }^{9}$

The uniform solution $u=\left\{u^{S}\right\}_{S \subseteq N}$ is defined as follows: for each non-empty subset of agents $S \subseteq N$, each $b_{S} \in[0,1]^{s}$ and $i \in S$,

$$
u_{i}^{S}\left(b_{S}\right)= \begin{cases}\min \left\{b_{i}, \lambda\left(b_{S}\right)\right\} & \text { if } \sum_{j \in S} b_{j} \geq 1 \\ \max \left\{b_{i}, \nu\left(b_{S}\right)\right\} & \text { otherwise }\end{cases}
$$

where $\lambda\left(b_{S}\right)$ is the unique solution of the equation $\sum_{j \in S} \min \left\{b_{j}, \lambda\left(b_{S}\right)\right\}=1$ and $\nu\left(b_{S}\right)$ is the unique solution of the equation $\sum_{j \in S} \max \left\{b_{j}, \nu\left(b_{S}\right)\right\}=1$.

Whenever the sum of the best shares exceeds the unity, the solution gives everyone his best share as long as it is below a bound that is determined endogenously to satisfy the feasibility constraint that the sum of shares adds up to one. Analogously, when the sum of the best shares is below the unity, the solution gives everyone the maximum between his best share and a bound that is determined endogenously so that the sum of the shares adds up to one. The uniform solution is widely used for its good properties. Sprumont (1991) showed that the uniform solution is the only one satisfying simultaneously strategy-proofness, efficiency and anonymity.

Given a partition function $\mu$, we define the uniform rule as $U^{\mu}=(\mu, u)$.

\footnotetext{
${ }^{9}$ Observe that our algorithm is more general since it can be applied to any solution. If it converges, the outcome is a stable partition for the solution.
} 
The equal gains-losses solution $e=\left\{e^{S}\right\}_{S \subseteq N}$ is defined as follows: for each non-empty subset of agents $S \subseteq N$, each $b_{S} \in[0,1]^{s}$ and $i \in S$,

$$
e_{i}^{S}\left(b_{S}\right)= \begin{cases}b_{i}+\frac{1-\sum_{j \in S} b_{j}}{\# S} & \text { if } \sum_{j \in S} b_{j} \leq 1 \\ \max \left\{0, b_{i}-\lambda\left(b_{S}\right)\right\} & \text { otherwise }\end{cases}
$$

where $\lambda\left(b_{S}\right)$ is the unique solution of the equation $\sum_{j \in S} \max \left\{0, b_{j}-\lambda\left(b_{S}\right)\right\}=1$.

As its name suggests, the equal gains-losses solution shares the surplus or shortage of the demand of a coalition equally across all its members with the limitation that in case of excess demand, the solution should not assign a negative share to any agent.

Given a partition function $\mu$, define the Equal Gains-losses rule as $E^{\mu}=(\mu, e)$.

Conjecture 1 There exist partition functions $\mu^{u}$ and $\mu^{e}$ such that the uniform rule $\left(\mu^{u}, u\right)$ and the equal gains-losses rule $\left(\mu^{e}, e\right)$ are stable.

Fix a solution $f$ and a vector of best shares $b \in[0,1]^{s}$. The algorithm proceeds as follows. In Step 0 we start with the partition formed by all individual coalitions. The set $V$ represents the set of players that remain to be checked given a partition to guarantee stability. Each time a new partition is formed the set $V$ is reinitiated to $N$.

Given a partition $\pi$, in Step 1 we apply the solution $f$ to the partition $\pi$ and we select the agent whose best share is further away from the allotment assigned to him by the solution (if there are more than one, we select the one with smaller index). We denote this agent by $i_{0}$. The set $\mathcal{K}$ denotes the set of the indices of the coalitions in partition $\pi$. Whenever we select a new agent to check, this set is reinitiated to incorporate all the indices in the partitions (i.e. all the coalitions).

Given an agent $i_{0}$, a partition $\pi$, and the set of coalitions that remain to be checked, in Step 2, we find the coalition $S_{k_{0}}$, with $k_{0} \in \mathcal{K}$, that agent $i_{0}$ would most prefer to join (or a new individual coalition) and check if $i_{0}$ is strictly better off by joining the new coalition (again, if there are more than one such coalitions, we select the one with smallest index). If that was not the case, we discard $i_{0}$ and move to the next worse off agent (Step 1).

If $i_{0}$ strictly prefers to move to the new coalition, then we check whether the members of the receiving coalition would like to accept him. If this is the case, we modify the partition accordingly, set $V=N$ and start Step 1 again. If the new coalition does not want to admit $i_{0}$, we discard coalition $S_{k_{0}}$ and look for the next coalition $i_{0}$ would most prefer to join (Step 2). This process goes till no agent who would like to move is allowed to do so, and hence we have a stable partition.

\section{Algorithm:}

INPUT: A solution $f$ and a profile $b$.

STEP 0: $\pi=\{\{1\}, \ldots,\{n\}\}$ and $V=N$.

STEP 1:

If $V=\emptyset \quad$ then go to Step 3 .

Else, $\quad$ set $i_{0}=\min \left(\arg \max _{i \in V}\left|b_{i}-f_{i}(\pi, b)\right|\right)$ and let $\mathcal{K}=\{1, \ldots, K\}$ be the set of indeces of the partition $\pi$.

STEP 2: 


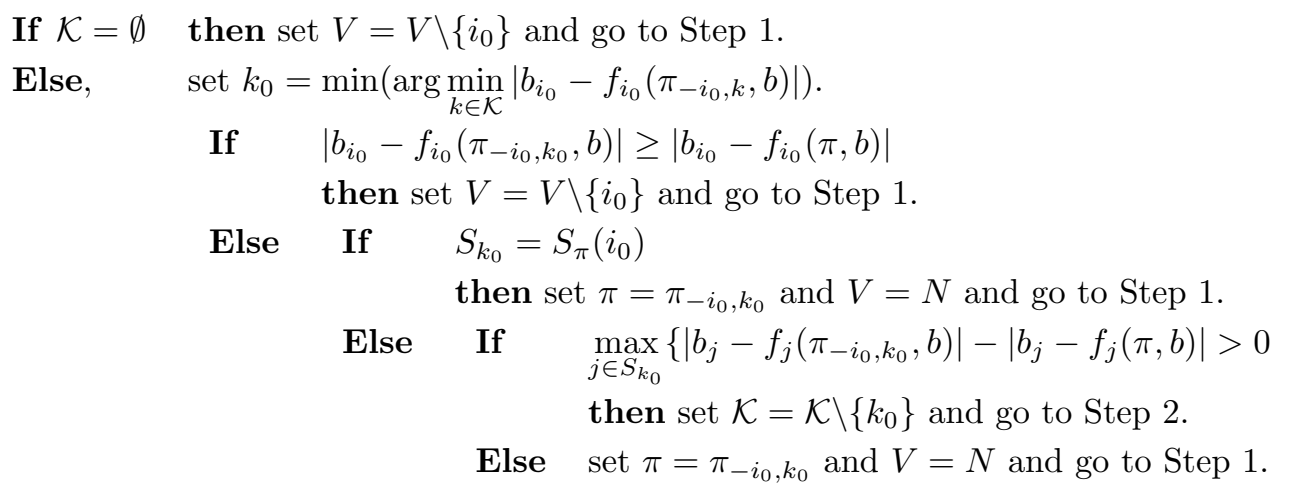

STEP 3:

No one who wants to move is allowed to do so.

The partition $\pi$ is stable at $b$ for $f$. END.

Examples 2 and 3 below show that for the uniform and the equal gains-losses rules stability is also incompatible with strategy-proofness, efficiency, anonymity and envy-freeness.

Example 2 Consider the uniform rule and assume $\mu^{u}$ is a partition function such that the rule $\left(\mu^{u}, u\right)$ is stable. Let $N=\{1,2,3\}$ and $b=(0.4,0.5,0.7)$. The only stable allocation at $b$ is $\left(\mu^{u}(b), u\left(\mu^{u}(b), b\right)\right)=(\{\{1,3\},\{2\}\},(0.4,1,0.6))$. Let $b_{2}^{\prime}=0.35$. Then, $\mu^{u}\left(b_{1}, b_{2}^{\prime}, b_{3}\right)=$ $\{\{1\},\{2,3\}\}$ and $u\left(\mu^{u}\left(b_{1}, b_{2}^{\prime}, b_{3}\right),\left(b_{1}, b_{2}^{\prime}, b_{3}\right)\right)=(1,0.35,0.65)$. Since $0.35 P_{2} 1,\left(\mu^{u}, u\right)$ is not strategyproof.

Let $N=\{1,2\}$ and $b=(0.4,0.9)$. The only stable allocation at $b$ is $(\{\{1\},\{2\}\},(1,1))$. However, the allocation $(\{\{1,2\}\},(0.1,0.9))$ is a Pareto improvement.

Finally, let $N=\{1,2,3\}$ and $b=(0.3,0.3,0.8)$. The are only two stable allocations at $b$, which are $(\{\{1,3\},\{2\}\},(0.3,1,0.7))$ and $(\{\{1\},\{2,3\}\},(1,0.3,0.7))$. But in none of them agent 1 and agent 2 receive the same shares and either agent 2 envies agent 1 or the opposite, so the uniform rule is neither anonymous nor envy-free.

Example 3 Consider the equal gains-losses rule and assume $\mu^{e}$ is a partition function such that the rule $\left(\mu^{e}, e\right)$ is stable. Let $N=\{1,2\}$ and $b=(0.4,0.9)$. The only stable allocation at $b$ is $\left(\mu^{e}(b), e\left(\mu^{e}(b), b\right)\right)=(\{\{1\},\{2\}\},(1,1))$. Let $b_{1}^{\prime}=0.1$. Then, $\mu^{e}\left(b_{1}^{\prime}, b_{2}\right)=\{\{1,2\}\}$ and $u\left(\mu^{e}\left(b_{1}^{\prime}, b_{2}\right),\left(b_{1}^{\prime}, b_{2}\right)\right)=(0.1,0.9)$. Since $0.1 P_{1} 1,\left(\mu^{e}, u\right)$ is not strategy-proof. Moreover, the allocation $(\{\{1,2\}\},(0.1,0.9))$ is a Pareto improvement over the stable allocation, so the rule is not efficient. Finally, let $N=\{1,2,3\}$ and $b=(0.3,0.3,0.9)$. The only stable allocations at $b$ are $(\{\{1,3\},\{2\}\},(0.2,1,0.8))$ and $(\{\{1\},\{2,3\}\},(1,0.2,0.8))$. But in none of them agent 1 and agent 2 receive the same shares and either agent 2 envies agent 1 or the opposite, so the equal gains-losses rule in neither anonymous nor envy-free.

\subsection{Efficiency}

Even though we have seen that stable rules are not necessarily efficient the following result holds. There exists a partition function $\mu^{e f}$ such that for any efficient solution $f$ the rule $\left(\mu^{e f}, f\right)$ is 
efficient. ${ }^{10}$ However, $\left(\mu^{e f}, f\right)$ may not be stable.

Proposition 3 There exists a partition function $\mu^{\text {ef }}$ such that for every efficient solution $f$ the rule $\left(\mu^{e f}, f\right)$ is efficient.

Proof Given $b \in[0,1]^{s}$ select arbitrarily a partition such that

$$
\mu^{e f}(b) \in \arg \min _{\substack{\pi \in \Pi \\ \pi=\left\{S_{1}, \ldots, S_{K}\right\}}} \sum_{k=1}^{K}\left|1-\sum_{i \in S_{k}} b_{i}\right|,
$$

and, from now on, denote it by $\pi=\left\{S_{1}, \ldots, S_{K}\right\}$. We check that the allocation $(\pi, x)$ is efficient at $b$, where $x=f(\pi, b)$.

First, notice that

$$
\sum_{k=1}^{K}\left|1-\sum_{i \in S_{k}} b_{i}\right|=\sum_{k=1}^{K}\left|\sum_{i \in S_{k}}\left(x_{i}-b_{i}\right)\right| .
$$

Since $f$ is an efficient solution, in every coalition $S_{k} \subseteq N, f^{S_{k}}\left(b_{S_{k}}\right)$ has the property that either (i) $f_{i}^{S_{k}}\left(b_{S_{k}}\right) \geq b_{i}$ for all $i \in S_{k}$ or else (ii) $f_{i}^{S_{k}}\left(b_{S_{k}}\right) \leq b_{i}$ for all $i \in S_{k}$. In particular, if the sum of the best shares is larger (or smaller) than 1 then, all agents in $S_{k}$ receive less (more) than their best shares. Thus, every $\left(x_{i}-b_{i}\right)$ has the same sign for all $i \in S_{k}$, implying that

$$
\left|\sum_{i \in S_{k}}\left(x_{i}-b_{i}\right)\right|=\sum_{i \in S_{k}}\left|x_{i}-b_{i}\right| .
$$

Therefore,

$$
\sum_{k=1}^{K}\left|1-\sum_{i \in S_{k}} b_{i}\right|=\sum_{k=1}^{K} \sum_{i \in S_{k}}\left|x_{i}-b_{i}\right|=\sum_{i \in N}\left|x_{i}-b_{i}\right| .
$$

We now proceed by contradiction. Suppose that $(x, \pi)$ is not efficient at $b$. Then, there exists an allocation $(\gamma, y)$ such that for all $i \in N,\left|y_{i}-b_{i}\right| \leq\left|x_{i}-b_{i}\right|$, and for at least one $j \in N$, $\left|y_{i}-b_{i}\right|<\left|x_{i}-b_{i}\right|$. Hence,

$$
\sum_{i \in N}\left|y_{i}-b_{i}\right|<\sum_{i \in N}\left|x_{i}-b_{i}\right|
$$

Without loss of generality we can assume that $y$ is Pareto optimal with respect to the partition $\gamma=\left\{S_{1}^{\prime}, \ldots, S_{K^{\prime}}^{\prime}\right\}$. Following the above reasoning,

$$
\sum_{k=1}^{K^{\prime}}\left|1-\sum_{i \in S_{k}^{\prime}} b_{i}\right|=\sum_{i \in N}\left|y_{i}-b_{i}\right|<\sum_{i \in N}\left|x_{i}-b_{i}\right|=\sum_{k=1}^{K^{\prime}}\left|1-\sum_{i \in S_{k}} b_{i}\right|,
$$

which contradicts the selection of $\pi$.

\footnotetext{
${ }^{10}$ Kar and Kibris (2008) have a similar result in a setting where the number of goods to be shared is fixed. In our case however, the number of goods, and hence the number of coalitions in the partition, is endogenous and depend on the preferences of the agents.
} 


\subsection{Symmetric Preferences}

First, without the symmetry assumption on preferences the complexity of the problem significantly increases. The reason is that then the associated partition function of a stable rule will require the use of much more information on the preference profile, than just the vector of best shares. For instance, let $n=2$ be the number of agents and let $b=(0.7,0.7)$ be the vector of best shares. Consider the proportional solution $p$. If single-peaked preferences are symmetric and the rule $(\mu, p)$ is stable then $\mu(0.7,0.7)=\{\{1,2\}\}$, because the share 0.5 is strictly preferred to 1 , which is not necessarily the case if preferences are not symmetric. Moreover, our results do not hold if the set of admissible single-peaked preferences are not symmetric. In particular, the proportional rule may not admit a partition function to constitute together a stable rule. To see that, let $n=3$ be the number of agents and consider the profile of single-peaked preferences $R=\left(R_{1}, R_{2}, R_{3}\right)$ depicted in Figure 1. Observe that agent 1's preferences are symmetric around $b_{1}=0.7$ while agents 2 and 3 ' preferences are not and have the property that $b_{2}=0.4$ and $b_{3}=0.5$.
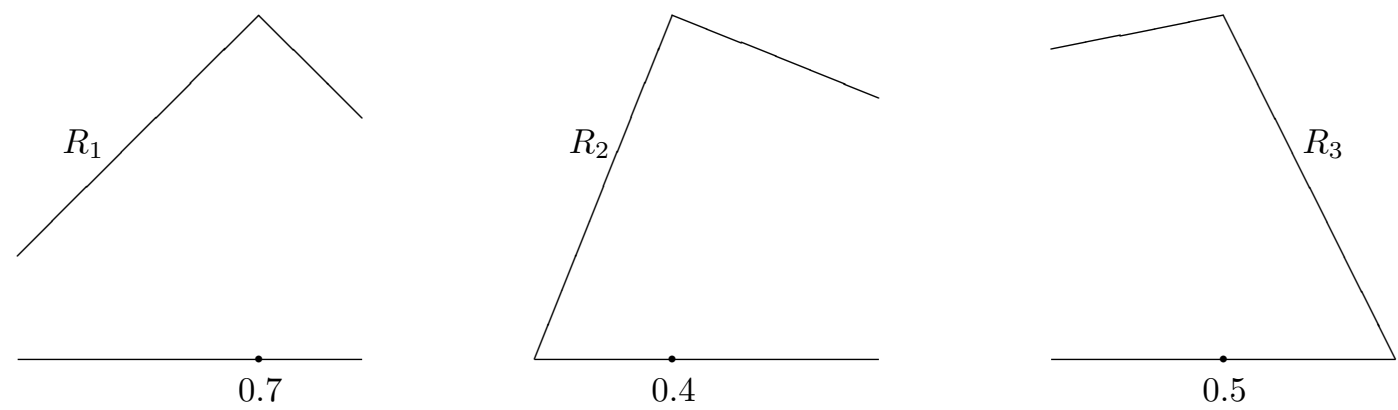

Figure 1

Observe that

- the partition $\{\{1,2\},\{3\}\}$ is not stable because 2 and 3 are better off together since $0 . \overline{4} P_{2} 0 . \overline{36}$ and $0 . \overline{5} P_{3} 1$;

- the partition $\{\{1,3\},\{2\}\}$ is not stable because 1 and 2 are better off together since $0 . \overline{6} \overline{3} P_{1} 0.58 \overline{3}$ and $0 . \overline{36} P_{2} 1$;

- the partition $\{\{1\},\{2,3\}\}$ is not stable because 1 and 3 are better off together since $0.58 \overline{3} P_{1} 1$ and $0.41 \overline{6} P_{3} 0 . \overline{5}$;

- the partition $\{\{1\},\{2\},\{3\}\}$ is not stable because 1 and 3 are better off together since $0.58 \overline{3} P_{1} 1$ and $0.41 \overline{6} P_{3} 1$; and

- the partition $\{\{1,2,3\}\}$ is not stable because 2 is better off alone since $1 P_{2} 0.25$.

A similar example can be exhibited for the sequential dictator solution. However, in many settings the hypothesis of symmetry seems natural since agents may evaluate equally loses and gains; for instance, when preferences are cardinal and the cost of moving away from the best share is equally perceived by the agent, independently on whether the movement is above or below the best share. 
Finally, the example also suggests that even under strong domain restrictions our results may not hold; for instance, under the domain of single-peaked preferences that are representable by utility functions which are linear on either of the best share, but that potentially have different slopes on the two sides.

\section{Acknowledgements}

We thank two referees of this journal for helpful comments. The work of G. Bergantiños is partially supported by research grant ECO2011-23460 from the Spanish Ministry of Science and Innovation and FEDER. J. Massó acknowledges financial support from the Spanish Ministry of Economy and Competitiveness, through the Severo Ochoa Programme for Centres of Excellence in R\&D (SEV-2011-0075) and through grant ECO2008-0475-FEDER (Grupo Consolidado-C), and from the Generalitat de Catalunya, through the prize "ICREA Academia" for excellence in research and grants SGR 2009-419 and 2014-515. The work of A. Neme is partially supported by the Universidad Nacional de San Luis, through grant 319502, and by the Consejo Nacional de Investigaciones Científicas y Técnicas (CONICET), through grant PIP 112-200801-00655. Part of this research was done while A. Neme visited the UAB thanks to the Generalitat de Catalunya grant SGR 2009-419.

\section{References}

[1] Adachi, T. (2010). "The Uniform Rule with Several Commodities: A Generalization of Sprumont's Characterization," Journal of Mathematical Economics 46, 952-964.

[2] Amorós, P. (2002). "Single-peaked Preferences with Several Commodities," Social Choice and Welfare 19, 57-67.

[3] Barberà, S., Jackson, M. (1995). "Strategy-proof Exchange," Econometrica 63, 51-87.

[4] Barberà, S., Jackson, M., Neme, A. (1997). "Strategy-proof Allotment Rules," Games and Economic Behavior 18, 1-21.

[5] Ching, S. (1992). "A Simple Characterization of the Uniform Rule," Economics Letters 40, $57-60$.

[6] Ching, S. (1994). "An Alternative Characterization of the Uniform Rule," Social Choice and Welfare 11, 131-136.

[7] Conley, J.P., Konichi, H. (2000). "Migration-proof Tiebout Equilibrium: Existence and Asymptotic Efficiency," Journal of Public Economics 86, 243-262.

[8] Dagan, N. (1996)."A Note on Thomson's Characterizations of the Uniform Rule," Journal of Economic Theory 69, 255-261.

[9] Ehlers, L. (2002a). "On Fixed-Path Rationing Methods," Journal of Economic Theory 106, $172-177$. 
[10] Ehlers,. L. (2002b). "Resource-monotonic Allocation when Preferences Are Single-peaked," Economic Theory 20, 113-131.

[11] Gensemer, S., Hong, L., Kelly, J. (1996). "Division Rules and Migration Equilibrium," Journal of Economic Theory 69, 104-116.

[12] Gensemer, S., Hong, L., Kelly, J. (1998). "Migration Disequilibrium and Specific Division Rules," Social Choice and Welfare 15, 201-209.

[13] Herrero, C., Villar, A. (2000). "An Alternative Characterization of the Equal-distance Rule for Allocation Problems with Single-peaked Preferences," Economics Letters 66, 311-317.

[14] Hylland, A., Zeckhauser, R. (1979). "The Efficient Allocation of Individuals to Positions," Journal of Political Economy 87, 293-314.

[15] Kar, A., Kibris, O. (2008). "Allocating Multiple Estates among Agents with Single-peaked Preferences," Social Choice and Welfare 31, 641-666.

[16] Massó, J., Moreno de Barreda, I. (2011). "On Strategy-proofness and Symmetric Singlepeakedness," Games and Economic Behavior 72, 467-484.

[17] Morimoto, S., Serizawa, S., Ching, S. (2013). "A Characterization of the Uniform Rule with Several Commodities and Agents," Social Choice and Welfare 40, 871-911.

[18] Moulin, H. (1980). "On Strategy-proofness and Single Peakedness," Public Choice 35, 437-455.

[19] Schummer, J., Thomson, W. (1997). "Two Derivations of the Uniform Rule and an Application to Bankruptcy," Economics Letters 55, 333-337.

[20] Sertel, M.R. (1992). "Membership Property Rights, Efficiency and Stability," Boğaziçi University Research Papers, Istanbul.

[21] Sönmez, T. (1994). "Consistency, Monotonicity, and the Uniform Rule," Economics Letters $46,229-235$.

[22] Sprumont, Y. (1991). "The Division Problem with Single-peaked Preferences: A Characterization of the Uniform Allocation Rule," Econometrica 59, 509-519.

[23] Thomson, W. (1994). "Consistent Solutions to the Problem of Fair Division when Preferences are Single-peaked," Journal of Economic Theory 63, 219-245.

[24] Thomson, W. (1995). "Population Monotonic Solutions to the Problem of Fair Division when Preferences are Single-peaked," Economic Theory 5, 229-246.

[25] Thomson, W. (1997). "The Replacement Principle in Economies with Single-peaked Preferences," Journal of Economic Theory 76, 145-168.

[26] Thomson, W. (2003). "Axiomatic and Game-theoretic Analysis of Bankruptcy and Taxation Problems: A Survey," Mathematical Social Sciences 45, 249-297. 